Doug Geisler, Eva K. Grebel, and Dante Minniti, eds.

\title{
New CCD Survey of Globular Clusters in M31
}

\author{
Myung Gyoon Lee, Sang Chul Kim \\ Astronomy Program, SEES, Seoul National University, Seoul, 151-742, \\ Korea
}

Doug Geisler, Juan Seguel

Departamento de Física, Grupo de Astronomía, Universidad de Concepción, Casilla 160-C, Concepción, Chile

\author{
Ata Sarajedini \\ Astronomy Department, University of Florida, Gainesville, FL \\ 32611-2055, USA \\ William Harris \\ Department of Physics and Astronomy, McMaster University, \\ Hamilton, ON L8S 4M1, Canada
}

\begin{abstract}
We present a progress report of our wide field CCD survey of globular clusters in M31. We have covered a $3 \mathrm{deg} \times 3 \mathrm{deg}$ area centered on M31, using the KPNO $0.9 \mathrm{~m}$ and Washington $C M T_{1}$ filters. Our survey is much deeper and more sensitive than previous surveys. We have found several hundred new globular cluster candidates in M31 in addition to confirming previously known globular clusters, and also have found a number of interlopers among previous globular cluster catalogs. We have also obtained spectra of about 500 objects among these candidates using HYDRA at the WIYN $3.5 \mathrm{~m}$ telescope, which are used for classification and measuring the radial velocity of the candidate objects. When completed, a new master catalog of globular clusters in M31 will be made, combining the new globular clusters with the known globular clusters.
\end{abstract}

\section{Introduction}

M31 is an ideal galaxy to study a globular cluster system in a spiral galaxy. There have been several surveys of globular clusters (GCs) in a large region of M31, but they were all based on photographic plates. There were a few surveys of globular clusters based on CCD observations as well, but they were limited to small areas (see Battistini et al. 1993, Mochejska et al. 1998, Barmby et al. 2000 and references therein). There are currently $\sim 800$ proposed GC candidates in M31 in the literature. Over 200 of these objects have been confirmed as GCs, 200 have been shown not to be clusters, and the nature of the remaining objects 
is as yet unknown (Barmby et al. 2000). Therefore, over the past few years, we have undertaken a wide field CCD survey of the globular clusters in M31.

\section{Observations and Data Reduction}

Using the KPNO $0.9 \mathrm{~m}$ and the Tek $2 \mathrm{k}$ CCD imager, we have obtained Washington $C M T_{1}$ images of $53\left(23^{\prime} \times 23^{\prime}\right)$ fields covering a region $3 \times 3 \mathrm{deg}^{2}$ centered on M31 to search for new GC candidates. Figure 1 illustrates a finding chart of M31 showing our survey region. Then using the WIYN $3.5 \mathrm{~m}$ and HYDRA (multifiber spectrograph) we have obtained spectra covering $3800 \AA-7000 \AA$ of about $500 \mathrm{GC}$ candidates. We have obtained the photometry of the point sources and the extended sources in the images using DOAPHOT II/ALLFRAME, and have reduced the HYDRA spectra using IRAF/DOHYDRA.

\section{Globular Cluster Search Methods}

We have selected globular cluster candidates using several criteria: a) colormagnitude diagrams, b) color-color diagrams, c) morphological classifiers based on the radial moments and the difference between the aperture magnitude and the point spread function fitting magnitude, and d) visual inspection of the images. Our recovery rate of the known bonafide GCs is estimated to be $>90 \%$. Finally we have used the spectra for confirming the GC candidates (see Seguel et al. 2002 as well).

\section{Results}

Our new photometric survey has produced a total of $\sim 1000$ new GC candidates with $T_{1}<20(V<20.5)$ mag. About 600 among these candidates are classified as class 1 (probable GC candidates) and class 2 (possible GC candidates), approximately doubling the number of good GC candidates. Figure 2 shows a color-magnitude diagram of sample new GC candidates as well as the known GCs, background galaxies and stars in one selected field (see Kim et al. 2002 for details). Note how successfully the known GCs are recovered and how many more new GC candidates (especially faint ones) are found in our new survey. Figure 3 displays the $T_{1}$ luminosity function and the $\left(C-T_{1}\right)$ color distribution of the new GC candidates. The luminosity function continues to increase with increasing magnitude, passing the expected turnover at $T_{1} \approx 16.7 \mathrm{mag}$. This shows that the faint end of the luminosity function $\left(T_{1}>18 \mathrm{mag}\right)$ contains many non-globular cluster objects. The color distribution of the GC candidates with $T_{1}<18-19$ mag shows a hint of bimodality, but needs further study to confirm it. Finally aperture photometry of the GC candidates will be used for further analysis.

However, these new GC candidates discovered as part of our photometric survey need to be confirmed by spectroscopy. As for the current spectroscopic data on the M31 GCs, Barmby et al. (2000) contains so far the most comprehensive catalog, which still only contains 200 velocity measurements and 188 




Figure 1. A finding chart of M31. The solid lines represent the boundaries of our globular cluster survey area, and the dotted lines represent the boundaries of planned, but not observed area.

spectroscopic metallicities of the GC candidates previously known. Spectroscopic data are being analyzed now. Figure 4 displays sample spectra of new spectroscopically confirmed GCs with $T_{1}=14.9-19.0$ mag. Several features typical for globular clusters ( $\mathrm{Ca}$ II $\mathrm{H}$ and $\mathrm{K}, \mathrm{H} \beta, \mathrm{G}$ band, Mgb complex, $\mathrm{Fe}$ $5270, \mathrm{Na} \mathrm{D}$, and $\mathrm{H} \alpha$ lines) are clearly noticed in the spectra. It is pleasing to find not only new GCs as faint as 19.0 mag but also new GCs as bright as 14.9 mag in our survey.

\section{Future Work}

We are planning to confirm spectroscopically most of the new GC candidates in M31. When our survey is finished, a new M31 GC catalog will be made, combining the new GCs and the known GCs. The final catalog of M31 globular clusters will be used: 1) to derive a reliable GC luminosity function for M31, the faint end of which was very incomplete in the previous data; 2) to investigate the bimodality of the metallicity distribution, which was seen only in the spectroscopic data of the previous catalog, but not clearly in any photometric data (Barmby et al. 2000); 3) to investigate the difference in mean luminosity between the metal-poor GCs and the metal-rich GCs. The present catalog of M31 globular clusters shows that the metal-poor GCs are 0.4 mag fainter than the metal-rich GCs (Barmby et al. 2000, Barmby 2002), which is the opposite to the case of the GCs in early-type galaxies and our Galaxy (Larsen et al. 2002). Our new catalog will be helpful to resolve this discrepancy; and 4) to investigate the systematic difference in kinematics between the metal-poor and the metalrich GCs, which provides critical information on the age difference between the two populations and strong constraints on galaxy formation scenarios.

Acknowledgments. This work was supported in part by the Korea Research Foundation Grant (KRF-2000-DP0450). 


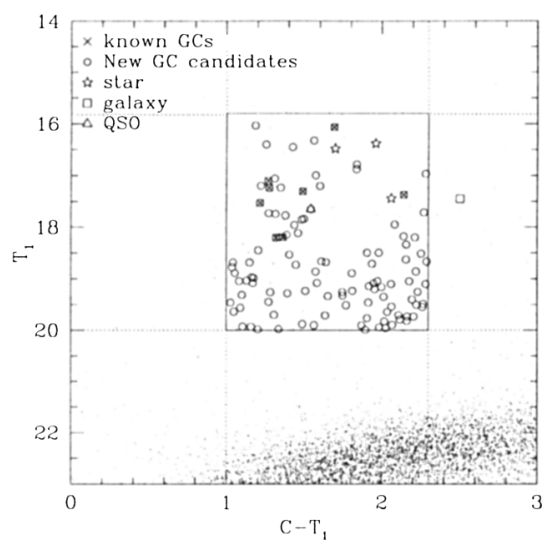

Figure 2. $\quad T_{1}$ vs $C-T_{1}$ color-magnitude diagram of the objects (dots) in one selected field in M31. The mass of faint red objects represents the red giant branch in M31. The $\times$ 's represent previously known globular clusters and the $\bigcirc$ 's are newly-discovered globular cluster candidates in seven of our fields in M31. The square and star symbols represent, respectively, a background galaxy and stars which were included in previous catalogs of globular cluster candidates in M31.
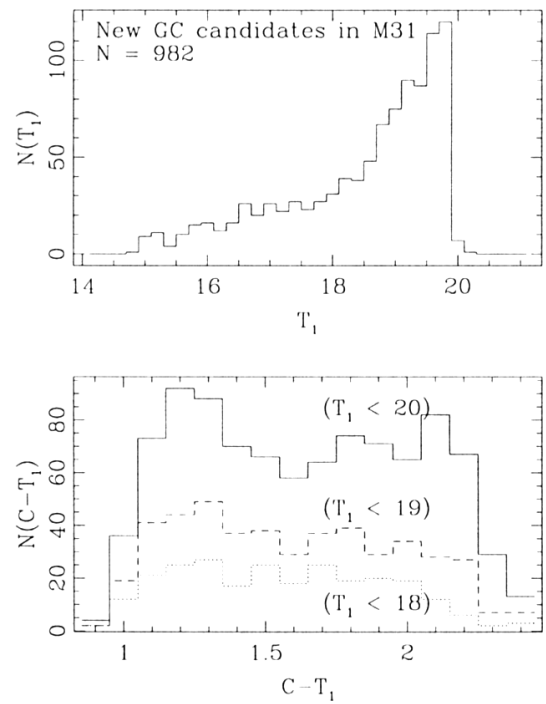

Figure 3. (Upper panel) $T_{1}$ luminosity function of the new globular cluster candidates. (Lower panel) $\left(C-T_{1}\right)$ color distribution of the new globular cluster candidates. 




Figure 4. Sample spectra of new globular clusters taken with WIYN+HYDRA in September 2000. Note the large range of magnitudes of the new globular clusters, $T_{1}=14.9-19.0$ mag.

\section{References}

Barmby, P., Huchra, J. P., Brodie, J. P., Forbes, D. A., Schroder, L. L., \& Grillmair, C. J. 2000, AJ, 119, 727

Barmby, P. 2002, this volume

Battistini, P. L., Bonoli, F., Casavecchi, M., Ciotti, L., Federici, L., \& Fusi Pecci, F. 1993, A\&A, 272, 77

Kim, S. C., Lee, M. G., Geisler, D., Seguel, J., Sarajedini, A., \& Harris, W. E. 2002 , this volume

Larsen, S. et al. 2002, this volume

Mochejska, B. J., Kaluzny, J, Krockenberger, M., Sasselov, D. D., \& Stanek, K. Z. 1998, Acta Astron., 48, 455

Seguel, J., Geisler, D., Kim, S. C., Lee, M. G., Sarajedini, A., \& Harris, W. E. 2002 , this volume 


\section{Discussion}

M. Kissler-Patig: How many new clusters did you find to date? How many new clusters do you expect to find and how does this influence the total number of known M31 clusters?

M. Lee: We have found about 1,000 globular cluster candidates from photometric data. We expect to find more than 100 genuine globular clusters finally, which should increase the total number of known M31 clusters by more than $20 \%$.

P. Barmby: What was the seeing for your observations and do you expect it to affect your results? Can you say anything about where in M31 your new cluster candidates are located?

M. Lee: Seeing was 1.5-2". Better seeing would have been better, but with what we had we could recover most of the known globulars. As for their location, most of the clusters I showed spectra for are in the outer parts of M31.

C. Gallart: Your color criteria would bias the results towards old clusters like those in the Milky Way. Can you comment?

M. Lee: At this moment we used the color criteria to search for genuine globular clusters. We also search for young populous clusters using a bluer color range. 